Revue interdisciplinaire sur la Grèce archaïque

24 | 2021

L'antropologo «classico» entre bêtes et dieux. Omaggi a Ezio Pellizer (Vol. 1)

\title{
Alcmeone e l'Alcmeonide: un altro Oreste?
}

Alcméon et l'Alcméonide : un autre Oreste?

Alcmeon and the Alcmeonis: Another Orestes?

\section{Carlo Brillante}

\section{(2) OpenEdition \\ Journals}

Edizione digitale

URL: https://journals.openedition.org/gaia/2265

DOI: $10.4000 /$ gaia.2265

ISSN: 2275-4776

\section{Editore}

UGA Éditions/Université Grenoble Alpes

\section{Edizione cartacea}

ISBN: 978-2-37747-292-5

ISSN: $1287-3349$

\section{Notizia bibliografica digitale}

Carlo Brillante, «Alcmeone e l'Alcmeonide: un altro Oreste?», Gaia [Online], 24 | 2021, online dal 31 mai 2021, consultato il 09 décembre 2021. URL: http://journals.openedition.org/gaia/2265 ; DOI: https:// doi.org/10.4000/gaia.2265

Questo documento è stato generato automaticamente il 9 décembre 2021.

Gaia. Revue interdisciplinaire sur la Grèce archaïque 


\title{
Alcmeone e l'Alcmeonide: un altro Oreste?
}

\author{
Alcméon et l'Alcméonide : un autre Oreste? \\ Alcmeon and the Alcmeonis: Another Orestes?
}

Carlo Brillante

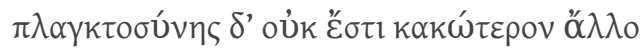

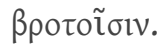

(Odissea, XV, 343)

1 Quale figlio di Amfiarao, il valente e sfortunato guerriero inghiottito dalla terra dopo la sconfitta argiva nella spedizione dei Sette, Alcmeone occupava un posto rilevante nell'epos degli Epigoni e un ruolo ancora maggiore aveva nell'Alcmeonide, un poema di cui conosciamo assai poco ma che probabilmente seguiva le vicende dell'eroe dalla seconda spedizione contro Tebe, coronata da successo, fino alle vicissitudini che lo portarono esule nelle lontane terre di Acarnania. La storia dell'eroe godette di un notevole successo anche nel teatro attico, come mostrano i titoli di tragedie che trattavano il medesimo tema. Soprattutto due tragedie di Euripide, l'Alcmeone a Psophis e l'Alcmeone a Corinto, traevano spunto dal matricidio e seguivano le vicende dell'eroe esule in varie regioni della Grecia. Una figura di primo piano nel mito, che aveva conquistato Tebe, compiendo un'impresa che non era riuscita alla precedente generazione dei Sette, che diventava matricida per volontà del padre e finiva i suoi giorni esule in terra straniera, non poteva sfuggire all'interesse dei tragediografi. La sua storia ripercorreva in fondo quella di un altro personaggio, forse meno coinvolto in imprese eroiche, ma ugualmente matricida ed esule: Oreste. Il confronto era già ben presente nella riflessione degli antichi ${ }^{1}$. Ma lo stesso interesse per la vicenda e per il personaggio hanno contribuito a rendere incerta la ricostruzione, sia pure a grandi linee, del contenuto dell'Alcmeonide. I testi giunti fino a noi tendono a presentare una storia uniforme, che spesso non distingue tra versioni diverse; di qui la necessità che narrazioni 'omogenee', come quelle della Biblioteca dello Pseudo-Apollodoro, di Diodoro e in parte quelle riportate da Pausania, siano usate con cautela nel ricostruire il contenuto dell'antico poema. È significativo, ad esempio, che nell'edizione di Bernabé, 
degli undici frammenti attribuibili all'Alcmeonide, ben quattro (frr. 8-11) siano compresi tra i dubia e che questi non siano riportati nell'edizione di Davies (1988) e in quella di West (2003). Un ulteriore fattore di rischio è rappresentato dal fatto che, secondo quanto tramandato, le vicende di Alcmeone erano narrate in almeno due poemi del ciclo epico: gli Epigoni e l'Alcmeonide. A meno che non si ritenga, con Welcker, che un unico poema fosse designato con nomi diversi ${ }^{2}$, si pone la necessità di individuare, per quanto è possibile, i temi trattati nel poema che aveva Alcmeone come protagonista. Beninteso, questo intervento non intende affrontare i vari problemi che pongono tuttora i magri resti dell'Alcmeonide, ma dovendo focalizzare l'attenzione sulle vicende dell'eroe che seguirono il matricidio - perché è su questa base che si pone il confronto con Oreste - non possiamo evitare di gettare uno sguardo sulle premesse generali dell'azione e su alcuni momenti della narrazione.

Oggetto principale del poema era probabilmente la vicenda personale di Alcmeone, variamente intrecciata con gli eventi che portarono la seconda spedizione argiva alla conquista di Tebe. Accanto a questo tema, condiviso con l'epos degli Epigoni, particolare rilevanza avevano gli eventi che costituivano il contenuto specifico del poema: il matricidio, cui seguiva la persecuzione delle Erinni, e l'esilio, che portò l'eroe a trovare una nuova sede in Acarnania, una terra lontana dalla sua terra di origine, situata ai limiti dell'ecumene ${ }^{3}$. Forse non è lontano dal vero il giudizio di Preller, che attribuiva all'Alcmeonide la medesima funzione che hanno i Nóøtor nell'epos troiano ${ }^{4}$.

3 Anche se non attestato da alcun frammento pervenutoci, è ragionevole ritenere che nel poema Alcmeone fosse il capo della seconda spedizione argiva. È quanto attesta la Biblioteca dello Pseudo-Apollodoro, che attribuisce all'eroe la vittoria nel duello con Laodamas, il figlio di Eteocle che difendeva la città e che precedentemente aveva ucciso Aigialeus, il figlio di Adrasto ${ }^{5}$. Questi eventi sono evocati nella profezia di Amfiarao secondo la versione pindarica della Pitica, VIII (vv. 44-47):

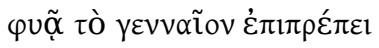

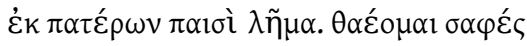

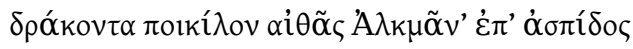

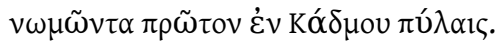

4 La profezia viene data quando la battaglia è in pieno svolgimento (v. 43: $\tilde{\omega} \delta$ ' $\varepsilon \tilde{i} \pi \varepsilon$ $\mu \alpha \rho v \alpha \mu \varepsilon ́ v(\omega v)$, forse nel luogo della scomparsa dell'eroe, dove in età storica sorgeva 1 A $A \varphi$ ó $_{\rho} \varepsilon_{10 v^{6}}$. Richiamando un momento decisivo del conflitto essa metteva in rilievo il valore di Alcmeone, che nelle visioni del padre si accinge a dare l'assalto decisivo alle mura di Tebe. Non è possibile stabilire se nel poema epico l'intervento di Amfiarao fosse stato sollecitato da un intervento del figlio in occasione dell'attacco alla città, secondo quanto suggerisce un altro frammento nel quale il parlante - probabilmente lo stesso Alcmeone - introduceva la preghiera con un appello alle divinità ctonie:

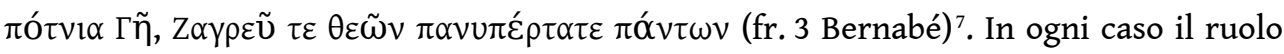
attribuito ad Alcmeone è tanto più interessante in quanto altri testi affermano che era Aigialeus a guidare la spedizione ${ }^{8}$. In questa seconda versione, nella quale il figlio ricopre il ruolo svolto dal genitore nella precedente generazione, è possibile scorgere con buon fondamento la versione degli Epigoni, secondo la proposta di Bethe: una differenza significativa rispetto all'Alcmeonide e probabile indizio di un diverso orientamento dei due poemi ${ }^{9}$. A un momento successivo alla presa di Tebe è forse da ricondurre il fr. 2 Bernabé, nel quale sono menzionate elaborate cerimonie funebri, probabilmente in onore dei caduti nel conflitto; è ragionevole ipotizzare che si trattasse 
dei guerrieri più illustri delle due parti: in primo luogo Aigialeus ucciso da Laodamas e lo stesso Laodamas caduto per mano di Alcmeone ${ }^{10}$.

5 Questi dati riferibili al conflitto tebano permettono di ritenere che la narrazione della presa di Tebe fosse parte integrante del poema e che in essa Alcmeone avesse un ruolo rilevante. Ma non dovevano essere questi gli elementi di maggior interesse del poema; essi erano comuni, sia pure con modalità diverse, all'epos degli Epigoni. Invece un ruolo centrale nell'Alcmeonide sembra fosse svolto dal matricidio e dalle conseguenze che ebbero sul protagonista. Anche su questo punto, peraltro, se si prescinde dalla testimonianza di autori che presentano una narrazione complessivamente omogenea (Pseudo-Apollodoro, Diodoro, Pausania), i dati pervenutici sono decisamente modesti. La sola testimonianza che menzioni il matricidio è offerta da uno scolio all'odissea, che cita Asclepiade di Tragilo e che Bernabé ha incluso tra i fragmenta dubia ${ }^{11}$. Dopo aver riassunto i precedenti della storia - la corruzione di Eriphyle, moglie di Amfiarao, da parte di Polinice e la conseguente partenza del marito per la guerra - il testo fa menzione dell'ordine che questi diede al figlio di non partire per la spedizione tebana prima di aver ucciso la madre. Consapevole del tradimento della sposa che di fatto gli imponeva di partecipare a una spedizione dalla quale non avrebbe fatto ritorno,

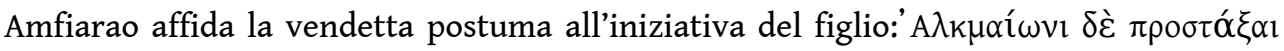

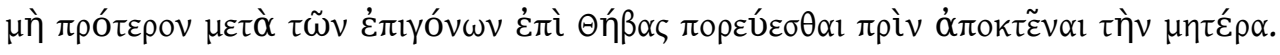

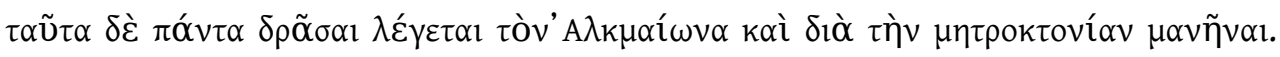

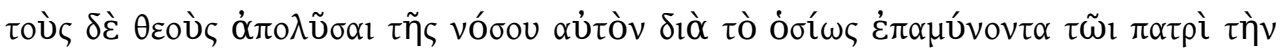

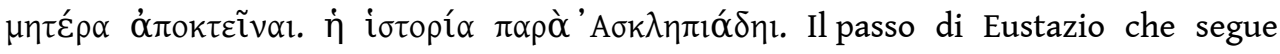
nell'edizione di Bernabé non aggiunge elementi rilevanti: Amfiarao impone al giovane di uccidere la madre al raggiungimento dell'età adulta e prima di partire per la guerra contro Tebe. L'eroe è infatti ancora fanciullo al tempo della prima spedizione tebana $\mathrm{e}$ dovranno trascorrere vari anni prima che dia compimento alla volontà paterna ${ }^{12}$. La $\mu \alpha v i ́ \alpha$ è qui comprensibilmente indotta dalle Erinni e in questo Alcmeone va

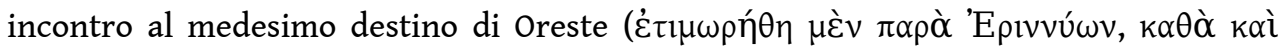

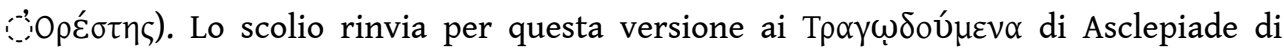
Tragilo, ma, dopo l'attenta analisi di Bethe, si è ritenuto, anche da altri studiosi, che Asclepiade utilizzasse una fonte epica e in particolare l'Alcmeonide ${ }^{13}$. Questo, che è il solo frammento attribuibile al poema che menzioni il matricidio, colloca l'evento prima della guerra degli Epigoni. La successione degli eventi è tuttavia controversa. Si è osservato che il matricidio provoca una contaminazione e l'intervento delle Erinni; sarebbe arduo immaginare che un personaggio in tale condizione potesse guidare un'impresa bellica ${ }^{14}$. Lo scolio citato offriva in verità una spiegazione di questa apparente anomalia: Alcmeone era stato liberato dalla vóбoৎ per un intervento degli dei, che giustificavano il suo comportamento in quanto aveva agito a sostegno del padre. Il problema della collocazione del matricidio prima o dopo la spedizione tebana fu puntualmente analizzato da Bethe. Egli distingueva due tradizioni della saga che faceva risalire ai due poemi epici che avevano trattato il tema: gli Epigoni e l'Alcmeonide ${ }^{15}$. Nella prima, Eriphyle sopravviveva alla distruzione di Tebe, come mostrerebbe il fatto che veniva corrotta una seconda volta da Thersandros, il figlio di Polinice, che faceva dono alla donna del peplo di Harmonia. Si tratta chiaramente della duplicazione di un motivo già presente nella Tebaide, dove il medesimo ruolo è svolto dalla collana di Harmonia ${ }^{16}$. Nella seconda invece Eriphyle era uccisa prima della spedizione, come afferma lo scolio citato all'odissea (fr. dub. 8 Bernabé), secondo il quale 
Amfiarao impose al figlio di non partire per la spedizione prima di aver ucciso la madre. Se quindi Alcmeone si atteneva all'ordine paterno, come si afferma nel medesimo frammento, il matricidio avvenne prima della spedizione. Bethe concludeva che nei due poemi i medesimi avvenimenti avessero uno svolgimento diverso e che nell'Alcmeonide, che ignorava l'episodio del peplo, il matricidio avvenisse prima della spedizione.

Queste argomentazioni possono apparire non decisive e si basano di fatto su una documentazione carente. Meritano tuttavia un approfondimento, in quanto valorizzano un elemento della storia non trascurabile. In particolare la seconda corruzione di Eriphyle a opera di Thersandros riprende un dato presente nella Tebaide $^{17}$. Può essere considerato senza difficoltà un motivo secondario, attribuibile a un'opera più recente che riprendeva un motivo ricorrente nell'epos più antico. L'ipotesi più ragionevole indirizza verso gli Epigoni, che si presenta come continuazione degli eventi narrati nella Tebaide ${ }^{18}$. Ma se Eriphyle era corrotta una seconda volta alla vigilia della seconda spedizione tebana, dovremo escludere che fosse uccisa dal figlio prima della sua partenza per la guerra. Il matricidio doveva avvenire dopo la conclusione del conflitto e la distruzione di Tebe. Su questo punto, beninteso, non disponiamo di attestazioni specifiche, ma il parallelismo richiede che il dono del peplo da parte di Thersandros abbia su Alcmeone le medesime conseguenze che il dono della collana aveva avuto sul padre Amfiarao. In entrambi i casi l'azione di persuasione esercitata su Eriphyle doveva avere come conseguenza la partenza dell'eroe. Nel caso di Alcmeone, pertanto, il matricidio doveva avvenire dopo il suo ritorno, che poi è quanto afferma lo Pseudo-Apollodoro. Questa successione degli eventi si inquadra abbastanza bene in un poema come gli Epigoni, che riprendeva e trattava con scarsa originalità eventi narrati nel poema più antico ${ }^{19}$. Altri poemi dovevano attenersi all'altra versione, che si distingueva per una maggiore linearità sul piano narrativo. Alcmeone seguiva l'ingiunzione del padre: raggiunta l'età adulta uccideva la madre, contraeva una vóøoৎ dalla quale era liberato per intervento degli dei e quindi partiva per la guerra. È questa in fondo la versione riportata dallo scolio citato all'odissea; essa presenta uno svolgimento lineare, evita la duplicazione di un motivo in rapporto alla figura di Eriphyle ed era forse presente nell'altro poema epico - l'Alcmeonide - che narrava i medesimi eventi.

Dopo il successo nella spedizione tebana, Alcmeone accompagnava Diomede in Etolia, impresa che aveva il fine di sostenere i diritti lesi di Oineus, il nonno di Diomede ${ }^{20}$. Questa seconda impresa, che precedeva di poco la spedizione troiana, non può essere considerata di origine recente, perché questi eventi sono presupposti dall'lliade, nel passo del catalogo delle navi che fa menzione del contingente degli Etoli ${ }^{21}$. Le ragioni della partecipazione di Alcmeone all'impresa vanno ricercate negli esiti provocati dal matricidio. La storia di Alcmeone è, sotto questo aspetto, particolarmente vicina a quella di Oreste. Che avvenga prima o dopo la spedizione degli Epigoni, il matricidio impedisce all'eroe di far ritorno ad Argo. L'invito a partecipare a un'impresa comune, che l'avrebbe portato lontano dalla terra di origine, appare quindi opportuno e ben accetto; e che la spedizione in Etolia con Diomede fosse inclusa nella narrazione è suggerito da un passo della Biblioteca dello Pseudo-Apollodoro, che riferisce come nell'Alcmeonide fosse narrata l'uccisione dei figli di Melas da parte di Tydeus ${ }^{22}$. Nella versione di Eforo Alcmeone, dopo la spedizione in Etolia, rifiutava, a differenza di Diomede, di seguire Agamennone a Troia e si poneva alla ricerca di una nuova sede 
nella quale insediarsi stabilmente. Probabilmente anche questo dato era compreso, come vedremo, nell'Alcmeonide.

Il matricidio fu in ogni caso determinante nel segnare le vicende successive dell'eroe. La vóбoৎ, che doveva assumere le forme di una $\mu \alpha v i ́ \alpha$, impedisce all'eroe di trovare una nuova terra che lo accolga. A essa fa riferimento lo scolio all'odissea che abbiamo

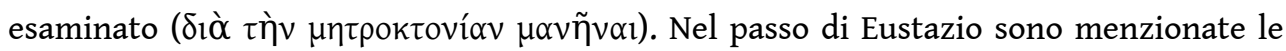
Erinni, con esplicito richiamo alla storia di Oreste. E non vi è dubbio che Alcmeone fosse soggetto, in conseguenza dell'atto compiuto, sia alla contaminazione sia alla follia. I due esiti non sono, tuttavia, sempre sovrapponibili. Lo scolio in fondo potrebbe aver riassunto in poche righe una vicenda più complessa. L'epos conosce riti di purificazione, necessari quando il soggetto si è macchiato di omicidio. Nell'Etiopide Achille, che aveva ucciso Tersite, deve recarsi a Lesbo, sacrificare ad Apollo, Artemide e Leto, ed essere purificato da Odisseo ${ }^{23}$. Per l'eroe non sono previste altre conseguenze. Il caso di Alcmeone è tuttavia più complesso: egli ha versato il sangue della madre, un consanguineo particolarmente vicino, e ha provocato l'inevitabile reazione dell'Erinni, cui è imputabile l'insorgere della $\mu \alpha v i ́ \alpha$. Nella versione dello Pseudo-Apollodoro, dopo la presa di Tebe, Alcmeone deve vagare, inseguito dall'erinni della madre ( $\mu \varepsilon \tau \tilde{\eta} \lambda \theta \varepsilon v$

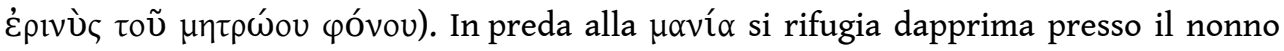
Oikles, quindi a Psofide in Arcadia. Qui è purificato dal sovrano locale Phegeus, di cui sposa la figlia Arsinoe; ma la persecuzione non cessa; a causa della sterilità della terra egli consulta l'oracolo, che gli ordina di recarsi alle rive dell'Acheloo ${ }^{24}$. Non sappiamo se questi eventi fossero narrati nell'Alcmeonide, ma questa successione mostra a sufficienza come la purificazione, pure compiuta con la richiesta ritualità, non fosse sufficiente a escludere la persecuzione delle Erinni. Il termine di confronto più ovvio è offerto dalla storia di Oreste. Nelle Eumenidi il personaggio si dichiara più volte esente da impurità e ottiene su questo punto l'assenso di Apollo, ma questo non basta a far cessare l'attacco delle Erinni ${ }^{25}$. Non sappiamo se un esito simile ricorresse anche nell'Alcmeonide, ma è un'ipotesi da prendere in considerazione se si pensa alle affinità che accomunano i due personaggi: entrambi eroi alle soglie dell'età adulta, si trovano nella condizione di dover compiere un atto di estrema gravità quale è il matricidio per compensare la colpa della madre verso il genitore; entrambi perseguitati dalle Erinni, sono costretti a lasciare la loro città per trovare una nuova sede in una terra lontana. Va inoltre osservato che, almeno nella poesia epica, l'intervento delle Erinni non compromette totalmente le capacità del soggetto e la sua posizione nella comunità. Nella Nekyia è ricordata la storia di Edipo, l'eroe parricida che sposò la propria madre. L'inevitabile intervento delle Erinni, procurò all'eroe molti dolori (Odissea, XI, 279 sg.),

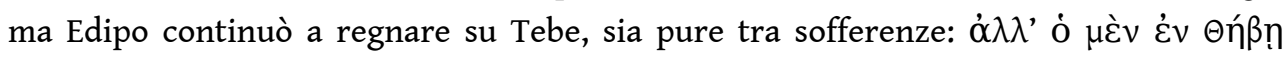

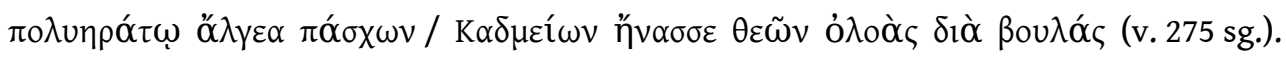
Nella versione iliadica del mito di Meleagro la maledizione lanciata dalla madre provoca l'intervento delle Erinni e il risentimento del giovane, che si rifiuta di difendere la città assediata dai Cureti, nonostante le suppliche degli abitanti e del padre Oineus. La maledizione non gli impedisce, tuttavia, di restare nelle case e di intervenire nel momento di estremo pericolo per la città, cedendo alle suppliche della sposa ${ }^{26}$. Va infine osservato che l'intervento delle Erinni non è sempre immediato. Se Edipo ne è vittima appena gli dei resero noto l'accaduto fra gli uomini (Od., XI, 274), Alcmeone, nella versione della Biblioteca, abbandonata la terra di origine, ha il tempo di rifugiarsi presso Phegeus, di essere purificato e di sposarne la figlia; solo più tardi la sterilità della terra gli imporrà di cercare una nuova sede ${ }^{27}$. In fondo anche l'inizio dell'Edipo re 
presenta l'immagine di un sovrano ormai stabilmente insediato sul trono di Tebe, senza che debba subire le conseguenze della propria condizione. Anche alla luce di queste considerazioni non vi sono ragioni sufficienti per dubitare che Alcmeone, aderendo alla richiesta del genitore, compisse il matricidio prima della partenza per la guerra, fosse purificato per volontà degli dei (l'estrema concisione dello scolio non permette di approfondire questo punto), ma che gli fosse impedito di tornare ad Argo forse (o anche) per l'intervento delle Erinni. Di qui la necessità di cercare una sede nuova, che fosse disposta ad accoglierlo consentendogli di dare inizio a una nuova vita.

Per questa seconda fase della storia, che doveva avere un ruolo cospicuo nell'Alcmeonide, disponiamo di varie testimonianze, che vedono l'eroe spostarsi in varie regioni della Grecia, con un seguito di avventure talvolta romanzesche. Un ruolo importante ebbero in questo ripensamento e progressivo arricchimento della vicenda mitica le versioni tragiche, che mettevano a frutto anche le tradizioni locali. Conosciamo meglio le versioni delle due tragedie euripidee - Alcmeone a Psophis e Alcmeone a Corinto - ma il tema fu trattato anche da Eschilo e da Sofocle ${ }^{28}$. I dati noti dalla tradizione mitografica vanno quindi trattati con cautela. Siamo tuttavia certi che le vicende successive alla vicenda tebana avevano un ruolo cospicuo anche nell'Alcmeonide. Ne offrono testimonianza i frammenti 4 e 5 dell'edizione di Bernabé. Il primo è riferibile a un contesto che narrava la spedizione di Diomede in Etolia, impresa eroica da collocare tra la spedizione degli Epigoni e la guerra di Troia. Si fa menzione, infatti, dell'uccisione dei figli di Melas, caduti nella generazione precedente per mano di Tydeus, il padre di Diomede ${ }^{29}$. Il puntuale richiamo all'Alcmeonide fa ritenere che nel poema Diomede rinnovasse l'impresa paterna recandosi in Etolia per difendere i diritti del nonno Oineus, questa volta defraudato del regno dai figli di Agrios $^{30}$. Il frammento 5 menziona i due figli di Ikarios, Alyzeus e Leukadios, che avrebbero regnato in Acarnania insieme con il padre. Alyzeus è l'eroe eponimo di Alyzeia, città dell'Acarnania, Leukadios è l'eponimo della colonia corinzia di Leukas. Il dato fornisce un'importante indicazione cronologica, in quanto permette di datare il poema a un'età successiva alla fondazione della colonia, risalente all'età dei Cipselidi. L'Alcmeonide è quindi un epos 'recente', che può risalire ai primi decenni del secolo $\mathrm{VI}^{31}$.

10 I dati ricavabili dai testi discussi permettono di concludere che l'abbandono della terra di origine, la purificazione e l'insediamento in una regione lontana da quella dove era avvenuto il crimine erano presenti già nell'Alcmeonide. In questa successione di eventi è possibile riconoscere una costellazione frequente in questo tipo di racconti ${ }^{32}$. Il soggetto contaminato dovrà essere accolto in uno spazio nuovo, senza passato $\mathrm{e}$ quindi senza confini definiti; dovrà separarsi definitivamente dalle precedenti esperienze, assumere una nuova identità e dare inizio a una fase nuova nella regione dove andrà a stabilirsi. Il personaggio che anche sotto questo aspetto presenta con Alcmeone i più significativi elementi di confronto è certamente Oreste. Anch'egli, inseguito dalle Erinni, deve abbandonare la città di origine, purificarsi e 'vagare' in numerose regioni della Grecia prima di giungere a una terra 'nuova' disposta ad accoglierlo. Non deve far ombra in tal senso il trattamento del mito nella tragedia di Eschilo. Questa segna un momento essenziale nella storia del mito, ma rappresenta anche un profondo e per molti aspetti originale ripensamento dell'antica vicenda. Se si considera l'ampio dossier mitico relativo al mito di Oreste i termini di confronto appariranno ancora più evidenti ${ }^{33}$. Dopo il matricidio, Alcmeone deve cercare una nuova sede. La partecipazione alla spedizione in Etolia insieme con Diomede, come la successiva rinuncia a partecipare alla guerra di Troia che si andava preparando in quel 
periodo, si spiegano bene in tale contesto. Il primo obiettivo dell'eroe è infatti quello di trovare una regione che lo ospiti. Non sappiamo dove giungesse nell'Alcmeonide, se ad esempio trovava accoglienza a Psofide, presso il re Phegeus, come accadeva nella tragedia euripidea ${ }^{34}$. Nella Biblioteca (III, 7, 5) si afferma che, fallito questo tentativo

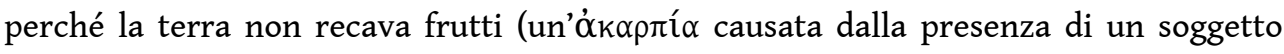
contaminato), egli interrogava l'oracolo e questo gli ordinava di recarsi presso l'Acheloo. Il testo che segue, probabilmente corrotto, non permette di seguire l'intero svolgimento della storia, anche se dovremo pensare a una seconda purificazione, questa volta ad opera di Acheloo, di cui Alcmeone sposava la figlia Kallirrhoe ${ }^{35}$. Per quanto possiamo giudicare dai dati pervenutici, l'eroe si attenne a questa indicazione: l'Acarnania fu la sua ultima sede. La medesima versione ricorre in Pausania, che aggiunge altri particolari. Finché si trovava tra gli Arcadi (Psofide) la vóøoৎ non gli dava tregua; Alcmeone si recò allora a Delfi per interrogare la Pizia. Questa profetizzò che il demone di Eriphyle avrebbe cessato di inseguirlo solo quando fosse giunto in una terra 'nuovissima', che il mare avesse fatto emergere dopo la contaminazione

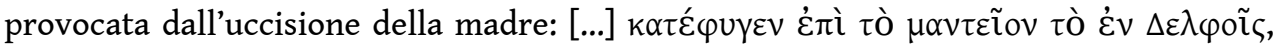

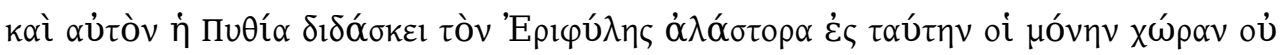

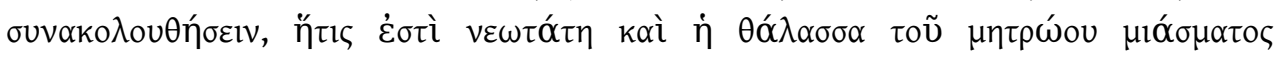

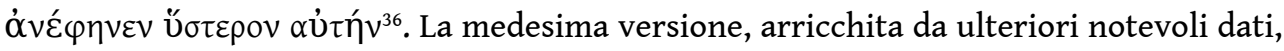
è ripresa in un notevole passo di Tucidide, che narra in qual modo Alcmeone prendesse possesso della nuova terra. Apollo gli aveva comunicato che non sarebbe stato liberato dai suoi 'timori' ( $\delta \varepsilon \hat{\imath} \mu \alpha \tau \alpha)$ prima di essersi insediato in una terra che, quando aveva ucciso la madre, non era vista dal sole e non era ancora terra, perché ogni altro luogo era contaminato per lui. L'eroe, dapprima in difficoltà per l'oscurità del responso, riconobbe poi che l'oracolo si riferiva agli accumuli alluvionali alla foce dell'Acheloo e ritenne che questa fosse la regione adatta nella quale insediarsi dopo il lungo vagare ${ }^{37}$ :

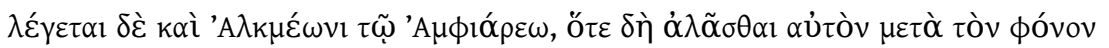

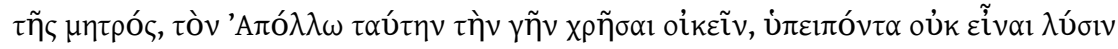

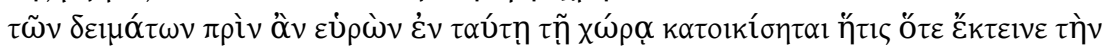

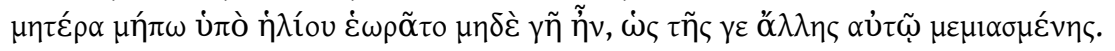

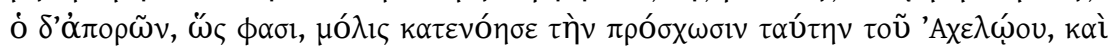

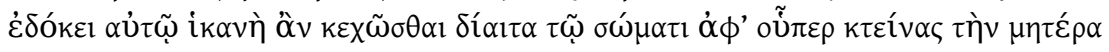

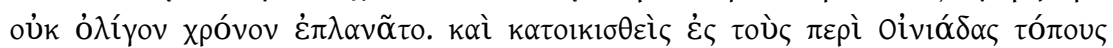

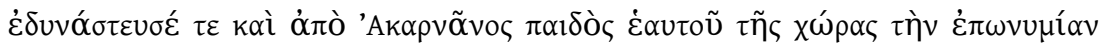

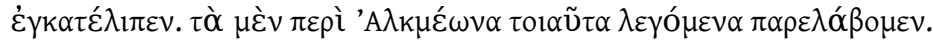

11 Il testo non fa menzione dell'Alcmeonide, ma si ritiene, con buon fondamento, che alla base del racconto fosse proprio questo poema, probabilmente attraverso la mediazione

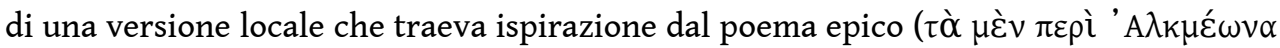
$\left.\tau o l \alpha \tilde{v} \tau \alpha \lambda \varepsilon \gamma o ́ \mu \varepsilon v \alpha \pi \alpha \rho \varepsilon \lambda \alpha \beta_{\beta} \mu \mu \varepsilon v\right)^{38}$. In questa versione è implicito l'intervento delle Erinni, richiamato dai $\delta \varepsilon i ́ \mu \alpha \tau \alpha$ che non danno tregua all'eroe e lo inseguono in qualunque regione si rechi; anche in questo il confronto con Oreste è puntuale. In tali condizioni l'intervento dell'oracolo diventa inevitabile, ma le condizioni poste dal dio sono apparentemente irrealizzabili. La richiesta muove da due presupposti: (1) che tutta la terra che ha assistito al matricidio sia per Alcmeone contaminata ( $\alpha \dot{v} \tau \tilde{\omega}$

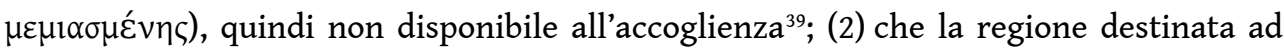
ospitarlo non sia mai stata raggiunta dal sole. L'unica via praticabile per rispettare tali condizioni impone di individuare una terra non soltanto nuova ma di fatto inesistente quando si compiva il matricidio. Non era sufficiente abbandonare Argo, perché tutta la 
terra era per lui contaminata. Il riferimento alla 'vista' del sole è in tal senso chiarificatore. Normalmente l'eroe esule si stabilisce in una terra lontana da quella di origine. Nel caso di Oreste questa necessità è ribadita dai numerosi racconti che narrano le vicende dell'eroe dopo il matricidio e lo fanno giungere in varie regioni della Grecia, dove la sua figura viene a integrarsi con culti e tradizioni locali. Ma ad Alcmeone questa via è preclusa: il sole illumina tutta la terra, quindi ogni regione gli sarà interdetta. Anche se il modello soggiacente è nei due casi il medesimo, nella vicenda di Alcmeone la richiesta di Apollo è tale da escludere l'eroe dall'intera ecumene $^{40}$. Essa impone di individuare una terra che sia talmente nuova da risultare inesistente anche in rapporto a un recente passato.

La versione tucididea valorizza quindi al massimo grado i rischi della contaminazione, che qui opera attraverso la visione. Se la terra 'nuova' non può essere contaminata da un evento al quale non ha assistito, il sole non risulterà contaminato dalla visione di una terra che non esisteva quando si compiva il matricidio. Quest'ultimo punto presuppone che il sole 'guardi' la terra nel momento stesso in cui l'illumina. Già in

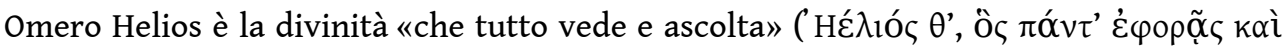

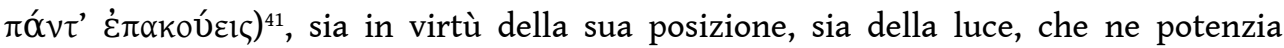
la capacità visiva. Nel canto XIV dell'Iliade la nube che avvolge l'amplesso di Zeus e Hera

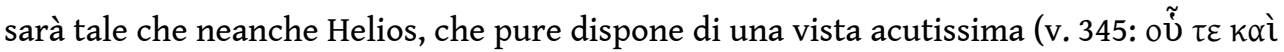

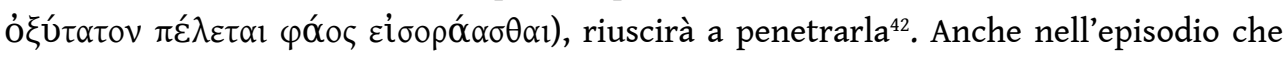
vede protagonista Alcmeone il rischio della contaminazione è presentato quale esito di un contatto messo in atto dalla visione. Su questo punto possiamo richiamare a confronto la conclusione dell'Edipo re. Di fronte a un personaggio di cui è venuta alla luce la tormentata vicenda, Creonte invita i servi ad avere riguardo per la luce del sole,

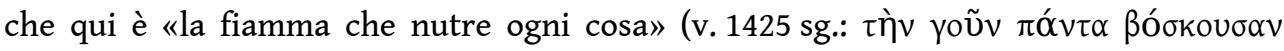

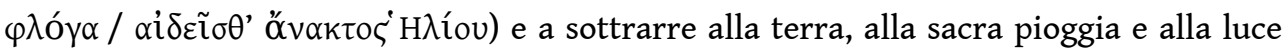

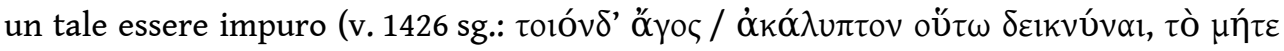

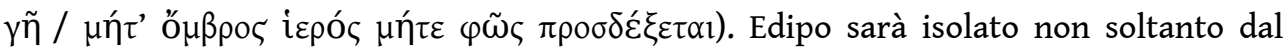
consorzio umano, ma anche dall'ambiente naturale, ugualmente esposto ai rischi della contaminazione. Il caso di Alcmeone si distingue da quello di Edipo perché il focus della narrazione si sposta dalla persona contaminata all'oggetto. La condizione dell'eroe matricida non è mutata, ma il contesto rinnovato fa sì che essa non sia più rilevante. Insediatosi ai limiti dell'ecumene, in una terra fino ad allora inesistente, Alcmeone troverà ospitalità perché non viene riconosciuto come matricida. Proprio come Oreste egli eviterà la persecuzione delle Erinni e potrà iniziare una nuova vita, ma rispettando condizioni più severe di quelle imposte al figlio di Agamennone. Il raggiungimento dell'ambita meta segna una frattura netta con il passato, conferisce al personaggio una nuova identità e gli consente di dare inizio a una nuova fase nella regione, come mostra l'intervento sanzionatorio finale con l'attribuzione del nome.

13 Concluderemo queste riflessioni con un altro breve frammento che, pur di contenuto diverso, richiama l'esito della vicenda di Alcmeone. Uno scolio all'Andromaca di Euripide riporta tre versi del poema nei quali è evocata l'uccisione di Phokos da parte dei suoi fratellastri Telamon e Peleus ${ }^{43}$ :

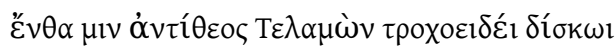

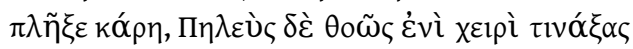

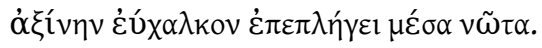


14

versione di Pausania, Telamon e Peleus furono costretti ad abbandonare Aigina. Più tardi Telamon cercò di farvi ritorno, negando di aver tramato la morte di Phokos. Ma il padre Aiakos non permise che sbarcasse nell'isola, e gli impose di parlare in propria difesa stando in piedi sulla nave oppure, se voleva, da un molo

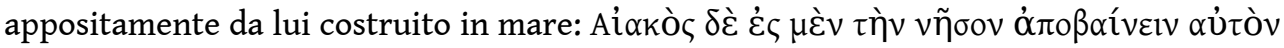

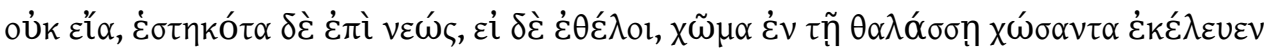

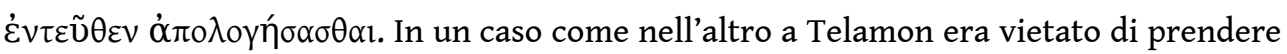
contatto con la terra dove era stato compiuto l'omicidio; può solo avvicinarsi restando in mare o parlando da una terra 'nuova' (il $\chi \tilde{\omega} \mu \alpha)$ che non aveva assistito all'omicidio ${ }^{44}$. Ritorna il tema che abbiamo incontrato in rapporto ad Alcmeone e al suo insediamento in Acarnania. Non sappiamo in quale occasione fosse introdotto questo mito (come exemplum in rapporto a personaggi di alcune generazioni precedenti?), ma è notevole che il medesimo tema ricorra due volte nel poema, sia pure con modalità diverse ${ }^{45}$.

Non vi è dubbio che le storie di Oreste e Alcmeone presentino convergenze significative. Punto di riferimento comune è certo il matricidio, con il quale si intende vendicare la morte proditoria del genitore; in entrambi i casi l'eroe si colloca dalla parte del padre e opera con il pieno consenso, se non con l'esplicita richiesta, della divinità. Il matricidio ha come conseguenza l'esclusione dalla comunità di origine e la ricerca di una nuova sede. Nel mito di Alcmeone questo tema conosce uno sviluppo particolare perché l'eroe può stabilirsi soltanto in una terra che sia totalmente 'nuova', come mostra la testimonianza di Tucidide. Altri aspetti non sono meno significativi: sia Oreste che Alcmeone ebbero un ruolo di primo piano nella tragedia, che contribuì non poco a ridefinirne la storia e il carattere, ed entrambi erano ben inseriti nella leggenda eroica dei Greci, anche se Oreste, a differenza di Alcmeone, non sembra distinguersi per la partecipazione a imprese eroiche di rilievo. In questo contributo si è cercato di ricuperare, nella misura modesta concessa da una documentazione carente, il ruolo di Alcmeone in un un poema epico tardo-arcaico che da lui prendeva nome. Per quanto possiamo giudicare erano narrati una serie di episodi, spesso eterogenei, che avevano come punto di riferimento la figura dell'eroe. Nella seconda spedizione contro Tebe Alcmeone aveva un ruolo di rilievo se era lui a guidare l'esercito argivo e uccideva di propria mano, in un confronto diretto, Laodamas, che aveva ereditato dal padre la sovranità di Tebe. Nella presentazione di questi episodi la versione dell'Alcmeonide si sovrapponeva - e su vari punti probabilmente si differenziava - da quella degli Epigoni, come abbiamo cercato di mostrare mettendo a confronto alcuni dati che sembrano riflettere orientamenti diversi del racconto.

\section{BIBLIOGRAFIA}

ANDERSEN Øivind, Thersites und Thoas vor Troia, «SO», 57, 1982, pp. 7-34.

BALLABRIGA Alain, Le soleil et le Tartare. L'image mythique du monde en Grèce archaïque, Paris, Éditions de l'EHESS, 1986.

Gaia, 24 | 2021 
BERNABÉ PAJARES Alberto, Fragmentos de Épica griega arcaica, Madrid, Editorial Gredos, 1979. BERNABÉ Albertus (ed.), Poetarum Epicorum Graecorum. Testimonia et Fragmenta. Pars I, Lipsiae, Teubner, $1996^{2}$.

BETHE Erich, Thebanische Heldenlieder, Leipzig, Hirzel, 1891.

BETHE Erich, s.v. Alkmaionis, Paulys Realencyclopädie der classischen Altertumwissenschaft, vol. I.2, Stuttgart, Metzlescher Verlag, 1894, coll. 1562-1564.

BREGLIA PULCI DORIA Luisa, Argo Amfilochia, l'Alkmaionis e la tradizione di Eforo, «AHS», 12, 1991-1994, pp. 123-140.

CINGANO Ettore, Epigonoi, in M. Fantuzzi \& C. Tsagalis (edd.), The Greek Epic Cycle and Its Ancient Reception. A Companion, Cambridge, Cambridge University Press, 2015, pp. 244-260.

DAVIES Malcolm, Epicorum Graecorum Fragmenta, Göttingen, Vandenhoeck \& Ruprecht, 1988.

DAVIES Malcolm, The Greek Epic Cycle, Bristol, Bristol Classical Press, 1989.

DEBIASI Andrea, Alcmeonis, in M. Fantuzzi \& C. Tsagalis (edd.), The Greek Epic Cycle and Its Ancient Reception. A Companion, Cambridge, Cambridge University Press, 2015, pp. 261-280.

DELCOURT Marie, Oreste et Alcméon. Étude sur la projection légendaire du matricide en Grèce, Paris, Les Belles Lettres, 1959.

DETIENNE Marcel, Le doigt d'Oreste, «Systèmes de pensée en Afrique noire», 14, 1996, pp. 23-38.

DODDS Eric R., The Greeks and the Irrational, Berkeley / Los Angeles / London, University of California Press, 1951.

FANTASIA Ugo (ed.), Tucidide. La guerra del Peloponneso. Libro II, Pisa, ETS, 2003.

FGrHist = JACOBY Felix, Die Fragmente der griechischen Historiker, vol. 1a, Leiden, Brill, 1957.

FRAZER James G., Apollodorus. The Library, Cambridge (MA) / London, Harvard University Press, 1921.

GANTZ Timothy N., Early Greek Myth. A Guide to Literary and Artistic Sources, Baltimore / London, Johns Hopkins University Press, 1993.

GIANNINI Pietro, in B. Gentili, P. Angeli Bernardini, E. Cingano \& P. Giannini (edd.), Pindaro. Le Pitiche, Milano, Mondadori, 1995.

GIORDANO Manuela, Contamination et vengeance: pour une diachronie du miasma, «Mètis» 12, 2014, pp. 291-310.

HARRIS Edward M., Is Oedipus Guilty? Sophocles and Athenian Homicide Law, in E. M. Harris \& D. F. Leão, Law and Drama in Ancient Greece, London, Duckworth, 2010, pp. 122-146.

HITZIG Hermannus \& BLÜMNER Hugo, Des Pausanias Beschreibung von Griechenland, Leipzig, Reisland, 1907.

HUMBERT Jean, Syntaxe grecque, Paris, Klincksieck, 1960.

HUXLEY George L., Greek Epic Poetry from Eumelos to Panyassis, London, Harvard University Press, 1969.

IMMISCH Otto, Klaros. Forschungen über griechische Stiftungssagen, «Jahrbuch für classische Philologie», Suppl. 17, 1890, pp. 125-210.

JOUAN François, «Les Corinthiens en Acarnanie et leurs prédécesseurs mythiques », in Mythe et politique (actes du colloque de Liège, 14-16 settembre 1989), Genève, Droz, 1990, pp. 155-166. 
KRAUSKOPF Ingrid, s.v. Alkmaion, in Lexicon Iconographicum Mythologiae Classicae, Zürich / München, Artemis Verlag, 1981a, pp. 546-552.

KRAUSKOPF Ingrid, s.v. Amphiaraos, in Lexicon Iconographicum Mythologiae Classicae, Zürich / München, Artemis Verlag, 1981b, pp. 691-713.

KULLMANN Wolfgang, Die Quellen der Ilias (Troischer Sagenkreis), Wiesbaden, Steiner Verlag, 1960.

LEGRAS Léon, Les Légendes thébaines dans l'épopée et la tragédie grecque, Paris, Société nouvelle de librairie et d'édition, 1905.

LONGO Oddone, La luce nell'ottica dei Greci, «Vichiana», 4, 1993, pp. 163-173 (= L'universo dei Greci. Attualità e distanze, Padova, Marsilio, 2000, pp. 228-238).

LULLI Laura, The Case of Telegony / Thesprotis. Some Notes about the Epichoric Aspects of a Cyclic Epic Poem, «Seminari Romani», n. s. 7, 2018, pp. 21-45.

MacDOWELL Douglas M., Athenian Homicide Law in the Age of the Orators, Manchester, 1963.

MOGGI Mauro \& OSANNA Massimo, Pausania. Guida della Grecia. Libro VIII: L’Arcadia, Milano, Mondadori, 2003.

MUGLER Charles, La lumière et la vision dans la poésie grecque, «REG», 73, 1960, pp. 40-72.

MUGLER Charles, Dictionnaire historique de la terminologie optique des Grecs, Paris, Klincksieck, 1964.

MUSTI Domenico \& BESCHI Luigi, Pausania. Guida della Grecia. Libro II: La Corinzia e l'Argolide, Milano, Mondadori, 1986.

OLIVIERI Oretta, Analogie e rovesciamenti tra i Sette e gli Epigoni, in P. Angeli Bernardini (ed.), La città di Argo. Mito, storia, tradizioni poetiche, Roma, Ateneo, 2004, pp. 79-91.

OLIVIERI Oretta, La geografia mitica delle imprese di Alcmeone dalla poesia epica alla tragedia, in

E. Cingano (ed.), Tra panellenismo e tradizioni locali. Generi poetici e storiografia, Alessandria, Edizioni dell'Orso, 2010, pp. 299-314.

PARKER Robert, Miasma. Pollution and Purification in early Greek Religion, Oxford, Oxford Clarendon Press, 1983.

PELLIN Alessandra, Un epos ellenistico su papiro: l'epyllium Diomedis (fr. ep. adesp. 2 Powell = P. Berol. 10566), in G. Cresci Marrone \& A. Pistellato (edd.), Studi in onore di Fulviomario Broilo (atti convegno di Venezia, 14-15 ottobre 2005), Padova, Sargon, 2007, pp. 471-485.

PRELLER Ludwig, Griechische Mythologie, vol. II, Berlin, Weidmann, 1861.

PRINZ Friedrich, Gründungsmythen und Sagenchronologie, «Zetemata», 72, München, Beck, 1979.

PUCCI Luca, Fuori di Atene. Miti e tradizioni su Oreste in Grecia antica, Canterano, Aracne, 2017.

RIZZINI Ilaria, L'occhio parlante. Per una semiotica dello sguardo nel mondo antico, Venezia, Istituto Veneto di Scienze Lettere e Arti, 1998.

ROBERT Carl, De Apollodori Bibliotheca (diss.), Berolini, typis expressit Gustavus Schade, 1873.

ROBERT Carl, Die Griechische Heldensage, vol. 3: Die Grossen Heldenepen; I. Abteilung, Die Argonauten; Der Thebanische Kreis, Berlin, Weidmann, 1921.

SCARPI Paolo (ed.), Apollodoro. I miti greci, traduzione di M. G. Ciani, Milano, Mondadori, 1996. SCHROEDER Otto, Pindars Pythien, Leipzig / Berlin, Teubner, 1922.

SEVERYNS Albert, L'Éthiopide d'Arctinos et la question du cycle épique, «Revue de philologie, de littérature et d'histoire anciennes», 49, 1925, pp. 153-183. 
SEVERYNS Albert, Le cycle épique dans l'école d'Aristarque, Liège / Paris, Carmanne / Champion, 1928.

SMALL Jocelyn P., A Matricide of Alcmaeon, «Mitt. deutsch. arch. Inst. Römische Abteilung» 83, 1976, pp. 113-144.

SOMMERSTEIN Alan (ed.), Aeschylus. Eumenides, Cambridge, Cambridge University Press, 1989.

TAPLIN Oliver, The Stagecraft of Aeschylus, Oxford, Oxford Clarendon Press, 1977.

VISSER Edzard, Homers Katalog der Schiffe, Stuttgart und Leipzig, Teubner, 1997.

WELCKER Friedrich Gottlieb, Der epische Cyclus oder die Homerischen Dichter, vol. 1, Bonn, Weber's Verlag, $1865^{2}$.

WELCKER Friedrich Gottlieb, Der epische Cyclus oder die Homerischen Dichter, vol. 2, Bonn, Weber's Verlag, $1882^{2}$.

WEST Martin Lichtfield, Greek Epic Fragments, Cambridge (MA) / London, 2003.

WESTLAKE Henry Dickinson, $\Lambda$ EГETAI in Thucydides, «Mnemosyne» 30, 1977, pp. 345-362.

WILAMOWITZ-MOELLENDORFF Ulrich von, Homerische Untersuchungen, Berlin, Weidmann, 1884.

ZERHOCH Sebastian, Erinys in Epos, Tragödie und Kult, Berlin, De Gruyter, 2015.

ZUNTZ Günther, Persephone. Three Essays on Religion and Thought in Magna Graecia, Oxford, Oxford Clarendon Press, 1971.

\section{NOTE}

1. Vedi Platone, Alcibiade secondo, $143 \mathrm{c}$; Eliano, Natura degli animali, I, 24; Suetonio, Nerone, 39. Nella Poetica di Aristotele (1453 a, 20), Alcmeone è ricordato, insieme con Oreste, tra i personaggi che hanno maggiormente ispirato i poeti tragici.

2. Welcker $(1865,195-196)$; per un esame dei contenuti Welcker (1882, 380-405). I temi specifici dell'Alcmeonide - come la lotta tra Tydeus e i figli di Melas, la storia dei figli di Ikarios (Alyzeus e Leukadios), la morte violenta di Phokos, l'agnello d'oro recato in dono ad Atreus - erano interpretate come narrazioni inserite nella trama generale $(1882,130)$. La tesi di un poema unico, designato indifferentemente con il nome di Epigoni o Alkmaionis, è stata ripresa da Prinz sulla base di una critica alle tesi di Bethe; un ruolo importante di mediazione, per la trasmissione di queste tradizioni epiche, è attribuito a un compendio mitologico con varianti $(1979,172-187)$.

3. Per una presentazione e analisi dei temi trattati nel poema, sui quali esiste un'ampia letteratura, si veda principalmente Bethe (1891, 126-140); Bethe (1894), con le osservazioni di Legras (1905, 88-109); Robert (1921, 956-967); Delcourt (1959, 31-54); Huxley (1969, 51-54); Prinz (1979, 174-187); Bernabé Pajares (1979, 71-89); Jouan (1990); Gantz (1993, 522-528); Olivieri (2010, 302-307); West (2003, 10-11); Debiasi (2015). Cingano dubita che nell'Alcmeonide fosse compresa anche la spedizione degli Epigoni (2015, 257-258).

4. Preller (1861). Più dubbia la tesi che attribuiva al poema il fine di stabilire un legame tra epos tebano e troiano, anche se di fatto svolgeva anche questa funzione: vedi Immisch $(1890,184)$; Severyns (1928, 224-225); Bernabé Pajares (1979, 81); Debiasi (2015, 273); Cingano (2015, 251-252). In ogni caso tali rapporti, che vedono protagonisti due successive generazioni di eroi, sono già presupposti dall'rliade (in primo luogo IV, 404-410).

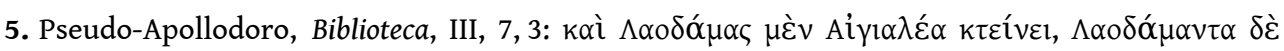
A $\lambda \kappa \mu \alpha i ́ \omega v$. Nella lista degli eroi che guidano la spedizione Alcmeone è nominato per primo: Pseudo-Apollodoro, Biblioteca, III 7, 2; Diodoro Siculo, IV, 66, 1; Bethe (1891, 109); Bethe (1894, 
1563); Debiasi (2015, 263 s.); Prinz valorizza la concordanza di Pindaro con le versioni della Biblioteca e di Diodoro $(1979,175)$.

6. Vedi Pausania, Descrizione della Grecia, IX 8, 3, da confrontare con il santuario menzionato da Erodoto (VIII, 134); Giannini (1995, ad vv.39-40). Probabilmente Pindaro riprende un tema trattato nell'epos: gli Epigoni, come riteneva Welcker (1882, 381); Rzach (1922, 2376), o più probabilmente l'Alcmeonide, dato il rilievo che qui assume il figlio di Amfiarao (Schroeder, 1922, 69).

7. Vedi in tal senso West $(2003,61$, n. 17), secondo il quale Alcmeone si rivolgeva alle divinità ctonie perché consentissero l'invio del padre Amfiarao, con riferimento a due brevi frammenti di Eschilo; nel primo Zagreus è identificato con Hades (fr. 5 Radt), nel secondo è detto suo figlio (fr. 228 Radt, con l'integrazione di Hermann). Questa versione del mito sarebbe solidale con quella della Pitica VIII, nella quale Amfiarao si rivolgeva al figlio in un momento decisivo del conflitto. Meno probabile un richiamo all'oracolo delfico (vedi Huxley, 1969, 52; Olivieri, 2010, 305; Debiasi, 2015, 270-271), sulla base della tradizione che collocava a Delfi, presso il tripode di Apollo, il corpo dilaniato di Dioniso: Callimaco frr. 43, 117; 643 Pfeiffer. Non sappiamo quando Zagreus, che nell'Alcmeonide era una divinità ctonia, fu identificato con Dioniso (vedi Zuntz, 1971, 81, n. 5). Inoltre l'invocazione non rientra nelle modalità consuete di interrogazione dell'oracolo, mentre risulta più appropriata se riferita alla richiesta di intervento del genitore del protagonista, che aveva dato prova del suo valore nel precedente conflitto. In tal senso indirizza anche il confronto con la testimonianza pindarica.

8. Vedi Euripide, Supplici, 1216; scolio a Iliade, 406 a Erbse, dove Aigialeus è nominato per primo; Bethe $(1891,110)$. Questa era forse la versione adottata ad Argo, come suggerisce un luogo di Pausania (Descrizione della Grecia, II, 20,5), che ricorda, accanto alle statue dei Sette situate nell'agora, quelle degli Epigoni; qui il personaggio menzionato per primo è Aigialeus (versione degli Epigoni). Nel monumento dedicato dagli Argivi a Delfi sono nominati per primi Stenelo e Alcmeone (Pausania, X, 10,5). Secondo una terza versione Aigialeus non periva nel conflitto, ma trovava rifugio, con alcuni Tebani, in Illiria, nel paese degli Enchelei: vedi Erodoto, V, 61, 2; Pausania, IX, 5, 13; 8, 6; 9, 5 .

9. Vedi Bethe (1891, 117-118 e 122); cfr. Legras (1905, 92-94). Per un diverso giudizio, che esclude la presenza di due versioni, riconducibili a poemi diversi, secondo la ricostruzione di Bethe, vedi Prinz (1979, 172-174).

10. Vedi in tal senso Huxley $(1969,53)$; Debiasi $(2015,268)$.

11. Asclepiade di Tragilo, FGrHist 12 F 29 = fr. ${ }^{\circ} 8$ Bernabé (= scolio a Odissea, XI, 326; Eustazio a Odissea, 1689, 10). Il frammento è stato attribuito all'Alcmeonide da Bethe (1891, 131; 1894, 1563); con qualche riserva, da Robert (1921, 956-957); così anche Severyns (1928, 227-228). Si veda anche

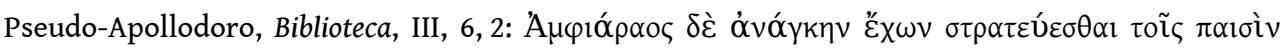

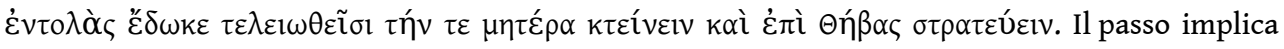
che la seconda spedizione contro Tebe fosse successiva al matricidio. Robert riteneva che su

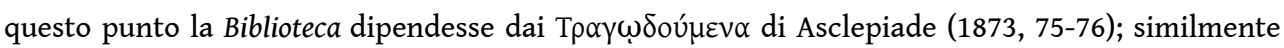
Bethe, secondo il quale entrambi i luoghi deriverebbero da un manuale mitologico (1891, 50-52). L'uccisione di Eriphyle era rappresentata già in un'anfora tirrenica del secondo quarto del VI secolo: vedi Small (1976, 124-126, che opportunamente esclude altre interpretazioni); Krauskopf (1981a, n. 3); Zerhoch (2015, 227, n. 35). Eriphyle è rappresentata riversa su un tumulo; dietro di essa, l'enorme serpente che si volge con la gola spalancata contro un guerriero che fugge sul carro rappresenta probabilmente l'Erinni dell'ucciso.

12. Secondo lo Pseudo-Apollodoro trascorsero 10 anni (Biblioteca, III, 7, 2). Sull'arca di Cipselo era raffigurata la partenza di Amfiarao, con Eriphyle, Amfiloco ancora infante in braccio alla nutrice e Alcmeone fanciullo (Pausania, Descrizione della Grecia, V, 17, 7). Per la fortuna del motivo nelle rappresentazioni figurate vedi Krauskopf (1981b, nn. 7-26). 
13. Vedi Bethe (1891, 128-130; cfr. 51, n. 12); Bethe (1894, 1563). Su questa linea Robert (1921, 956-957) e Severyns $(1928,228)$. Delcourt ha osservato che questa successione degli eventi, considerata la più naturale, conserva un tratto di arcaicità per il fatto che non menziona l'oracolo delfico e attribuisce la guarigione dell'eroe genericamente agli dei (1959, 46 e 51).

14. Dubbi su questa base esprimeva già Jacoby nel commento al frammento di Asclepiade (1957, 488-489). Richiamandosi a questo giudizio, Prinz escludeva che dallo scolio potesse ricavarsi una sequenza temporale (1979, 178-179); Krauskopf (1981a, 547); Gantz (1993, 525). Va notato peraltro che il giudizio di Jacoby è più sfumato: la purificazione a opera della divinità (e non di Phegeus o dell'Acheloo) poteva spiegare la successione degli eventi. In ogni caso queste riserve sembrano eccessive; lo scolio cita l'esplicita richiesta di Amfiarao e aggiunge che Alcmeone si attenne ad

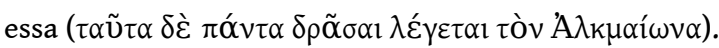

15. Bethe, supra, n. 13.

16. Non ci sono pervenuti frammenti del poema che menzionino l'episodio, ma ad esso si riferiscono due luoghi dell'Odissea (XI, 326-327; XV, 246-247). Un verso dell'Olimpica VI di Pindaro, nel quale Adrasto loda Amfiarao in occasione delle cerimonie funebri per i caduti a Tebe

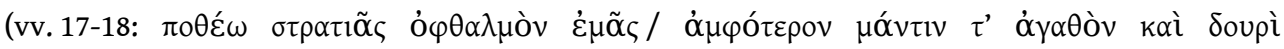
$\left.\mu \alpha \rho^{\rho} v \alpha \sigma \theta \alpha \mathrm{l}\right)$, che lo scolio dice ispirato alla Tebaide ciclica (a Olimpiche, VI, 26 = fr. 10 Bernabé), fa ritenere che del poema fosse parte anche la corruzione di Eriphyle. L'evento era centrale nel mito. Nella Tebaide era forse presente anche la disputa tra Amfiarao e Adrasto, che avrebbe portato, dopo il giudizio di Eriphyle, alla partenza dell'eroe: vedi scolio a Pindaro Nemee, IX, 30b (= fr. $7^{*}$ West): Bernabé Pajares (1979, 62-63); Davies (1989, 27); West (2003, 7).

17. V. supra, nota 16 .

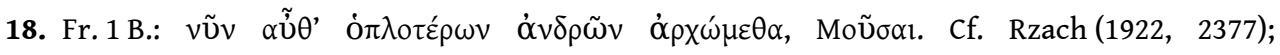
Severyns $(1925,176)$; West $(2003,9)$ : «the opening of the Epigoni [fr. 1] proclaims to be a continuation of the Thebaid»; Olivieri (2004); Cingano $(2015,247)$.

19. È improbabile che questa versione abbia avuto origine nella tragedia. Già Ellanico fa

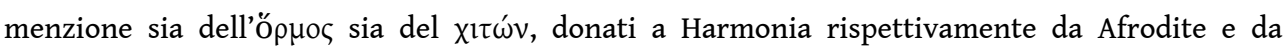
Athena: FGrHist 4 F 98 (=scolio a Euripide, Fenicie, 71); Bethe (1891, 129). Robert (1921, 957) ipotizza che sia stato lo stesso Ellanico a introdurre questo elemento nella saga, ma più probabilmente esso era già presente nella poesia epica.

20. Bethe $(1891,134-136)$; Bethe $(1894,1563)$. Talvolta la spedizione in Etolia è posta dopo la guerra di Troia (Antonino Liberale, Metamorfosi, 37; Igino, Miti, 175), secondo una rilettura tesa probabilmente a inquadrare l'evento tra i vóotor degli eroi. Su questo episodio, vedi infra, n. 30.

21. Iliade, II, 638-644; cf. XIV, 114-129; vedi Kullmann (1960, 102-103); Visser (1997, 599-601). Non vi è motivo di ritenere che l'Alcmeonide rielaborasse, integrandoli, spunti presenti nell' Iliade, come propone Andersen (1982, 10-13). Entrambi i poemi attingevano a un patrimonio comune in rapporto alle esigenze della narrazione.

22. Biblioteca, I, 8, 5 = Alcmeonide, fr. 4 B.; infra, § 9.

23. Vedi Etiopide, «Argomento», 68, 6-10 Bernabé. L'Alcmeonide potrebbe includere altri esempi di purificazione: quello di Peleus, che con il fratello Telamon provocò l'uccisione del fratellastro Phokos (fr. 1 B.), e di Pelope, uccisore di Mirtilo: vedi in tal senso Debiasi (2015, 273-275). Per un riesame del presunto 'silenzio omerico' sui rituali di purificazione, vedi Parker (1983, 130-143). Già Dodds (1951, 35-36) aveva osservato come «the difference between Homer and the Archaic Age is relative, not absolute; for it is a mistake to deny that a certain minimum of catharsis is practiced in both epics». Giordano $(2014,298)$ ritiene che non si possa risalire al di là della fine del secolo VII, età di composizione dell'Etiopide.

24. Pseudo-Apollodoro, Biblioteca, III, 7, 5.

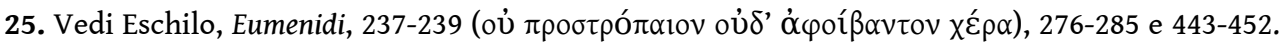
Oreste ottiene l'assenso di Apollo: vv. 576-578; Taplin (1977, 381-384); Sommerstein (1989, ad v. 237). Oreste è purificato da Apollo a Delfi: Parker (1983, 139, n. 142; 386-388); Zerhoch (2015, 
238, n. 82, con rinvii). Il fatto che nell'Odissea non si faccia menzione dell'intervento delle Erinni non è decisivo, perché il poema è interessato a presentare il personaggio come exemplum positivo (I, 29-31, 298 sg. e 306-310; IV, 546; XI, 461), anche se non manca un richiamo implicito al matricidio (III, 310).

26. Iliade, IX, 529-599; Pseudo-Apollodoro, Biblioteca, I, 8, 3.

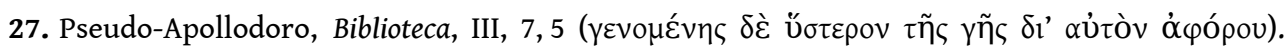
A una reazione non immediata delle Erinni fa pensare anche la sintesi di Igino (Miti, 73), sulla quale ha richiamato l'attenzione Immisch $(1890,181)$ : Alcmaeon memor patris praecepti Eriphylem matrem suam interfecit; quem postea furiae exagitarunt.

28. Vedi Robert (1921, 959-960); Prinz (1979, 178-179); Gantz (1993, 523 e 526-527); Olivieri (2010, 307-309).

29. Pseudo-Apollodoro, Biblioteca, I, 8, 5 = fr. 4 B.

30. Pseudo-Apollodoro, Biblioteca, I, 8, 6. Eforo, FGrHist, 70 F 123ab (= fr. 9 Bernabé). Cfr. Pausania, Descrizione della Grecia, II, 25, 2. L'intervento di Diomede si inserisce in una faida per la regalità insorta tra i discendenti di Porthaon e che interessò due generazioni di eroi: cfr. Biblioteca, I, 7, 10; 8, 5 sg.; vedi anche Antonino Liberale, Metamorfosi, 37, 1; Igino, Miti, 175 (entrambi collocano l'evento dopo la guerra di Troia). La spedizione in Etolia era trattata nell'Oineus di Euripide, dove compagno di Diomede era Stenelo, quindi in un epos ellenistico, l'epyllium Diomedis: v. Collectanea Alexandrina, 72-76; Huxley (1969, 51, n. 1); Pellin (2007).

31. Wilamowitz proponeva una data intorno al 600 o di poco successiva $(1884,73$, n. 2); Immisch (1890, 187); Bethe (1891, 132); Bethe (1894, 1563); Debiasi pone come terminus post quem la metà del secolo VII e individua le origini del poema nell'ambiente corinzio dei Cipselidi (2015, 277-278); ma è stata proposta anche una datazione alla prima metà del secolo VI (Jouan 1990, 163), con la possibilità di giungere fino agli inizi del V (West 2003, 11, per la menzione di Zagreus: fr. 3 Bernabé). Va peraltro osservato che il nome di Leukas, riferito all'isola o alla regione, era forse più antico: vedi Kullmann (1960, 380-381), che rinvia a Odissea, XXIV, 377 sg. Qui la città di

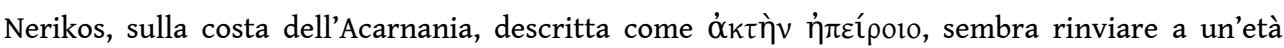
nella quale l'isola non era separata dal continente, a un'età cioè precedente lo scavo del canale ad opera dei Corinzi nell'età di Cipselo (Strabone, X, 2, 8 [452]). Fu in tale occasione che il nome di Leucade fu trasferito all'isola. Cfr. Tucidide, III, 7, 4 sg. In Iliade, IV, 491 Leukos è un compagno di Odisseo. Sull'importanza delle tradizioni locali nei poemi del ciclo epico, vedi da ultimo Lulli (2018), con altri rinvii.

32. Per le tradizioni sugli eroi che vanno incontro a un destino simile a quello di Oreste e Alcmeone vedi Parker (1983, 375-392: Appendix 7); per i rituali di purificazione in seguito a omicidio: Parker (1983, 370-374: Appendix 6). Sulla purificazione di Oreste vedi Delcourt (1959, 92-102). Detienne, in un interessante saggio dedicato all'eroe, rileva come intorno a Oreste si siano raccolte le tradizioni più significative sulle conseguenze provocate dal sangue versato (1996, 26-27).

33. Un'ampia rassegna che prende in esame le tradizioni sul personaggio estranee alla tragedia e che lo vedono esule e 'fondatore' in varie regioni della Grecia è offerta dal recente contributo di Pucci (2017).

34. Vedi Euripide, frr. 71 e $72 \mathrm{~K}$.

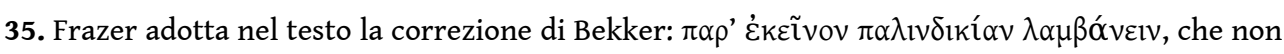
appare fondata, perché il tema del processo è estraneo al mito di Alcmeone. Verisimilmente si fa riferimento a una seconda purificazione ad opera di Acheloo (Bethe, 1891, 137). Più vicina al

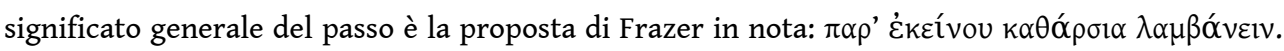
Per altre proposte di integrazione, vedi Scarpi (1996, ad loc.).

36. Pausania, Descrizione della Grecia, VIII, 24, 8 . Il contesto nel quale ricorre la narrazione, con la menzione del sepolcro di Alcmeone a Psofide (VIII, 24, 7), ha fatto ritenere che il racconto fosse 
parte di una tradizione locale: vedi Hitzig-Blümner (1907, ad loc.); Robert (1921, 959); Moggi \& Osanna (2003, ad loc.).

37. Tucidide, II, 102, $5 \mathrm{sg}$. ( $=$ fr. ${ }^{\circ} 10$ Bernabé). Il riferimento è alle isole Oiniadai (scolio a Tucidide, II, 102, 1), formatesi alla foce dell'Acheloo. Cfr. Erodoto, II, 10, 2 (formazione delle isole Echinadi). La versione di Tucidide è la più ampia sulla conclusione della storia di Alcmeone. Il riferimento ai

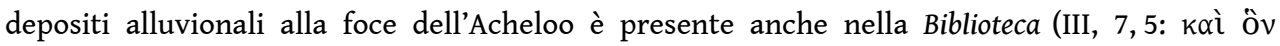

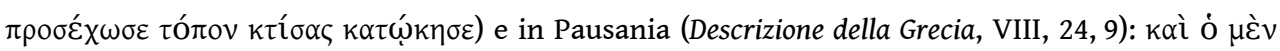

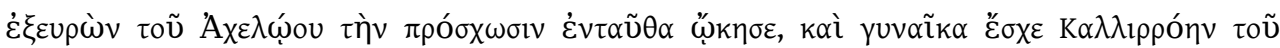

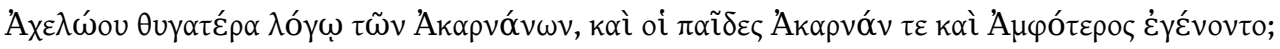

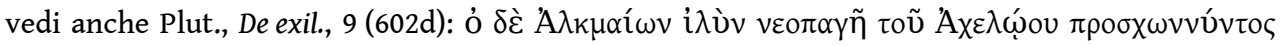

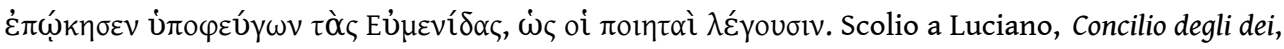
12 (212 sg. Rabe).

38. Per la tesi della derivazione dal poema epico vedi Bethe (1891, 138-139), che criticava giustamente la tesi di Immisch $(1890,187-188)$, di una dipendenza di Tucidide dalle versioni tragiche; Huxley (1969, 51), Krauskopf (1981a, 547); Breglia Pulci Doria (1991-1994, 131); West (2003, 11); Olivieri (2010, 306); Debiasi (2015, 273-274). Meno probabile un richiamo generico, come ipotizza Westlake (1977, 358-359), che presuppone una conoscenza diffusa dell'episodio. La versione di Tucidide presenta tratti peculiari, come si cerca di mostrare nel

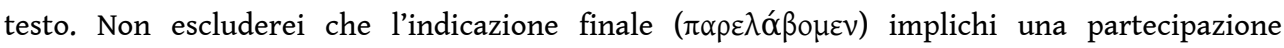
personale alla spedizione di Formione in Acarnania: vedi Fantasia (2003, 603, ad Thuc., II, 103).

39. La frase esprime l'idea che ogni altra terra era contaminata 'per lui' (Alcmeone). Il dativo non equivale a u̇đó con il genitivo, come talvolta si intende, ma è un dativo d'interesse: individua il soggetto per il quale la terra era contaminata (cfr. Humbert, 1960, 287). Solo Alcmeone era colpito dal divieto.

40. In questa narrazione l'Acheloo, posto nell'estremo occidente, segna il limite del mondo abitato. Significativa l'associazione con Oceano nell'Iliade (XXI, 194-197); Ballabriga (1986, 35-36 e 42-45).

41. Iliade, III, 277, nell'ambito di una formula di giuramento; vedi anche Odissea, XI, 109; XII, 323;

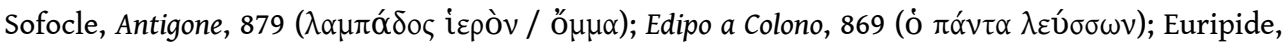
Ifigenia Taurica, 193 sg.; Ione, 1467. Si veda anche la definizione pitagorica degli occhi quali 'porte

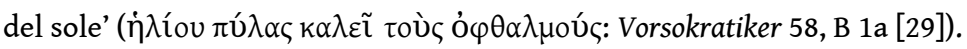

42. Per la visione quale esito della luce emessa attraverso i raggi e del sole quale entità fornita di una vista potente vedi Mugler (1960, 47-48 e 66-67) con ampia esemplificazione; Mugler (1964, 191-194); inoltre l'eccellente analisi dedicata al tema, anche in rapporto alla riflessione dei presocratici e del pensiero filosofico e scientifico, da Rizzini (1998, soprattutto 128-144); Longo (2000).

43. Scolio a Euripide, Andromaca, 687 (=fr. 1 Bernabé). Sul tema vedi Pausania, Descrizione della Grecia, II, 29, 9 sg.; Pseudo-Apollodoro, Biblioteca, III, 12,6; scolio a Pindaro, Nemee, V, 25a; Severyns (1928, 234-236); Huxley (1969, 52-53).

44. Le condizioni poste da Aiakos richiamano le modalità previste per l'omicidio involontario che ad Atene era celebrato nella località di Freatto (probabilmente a Zea, porto del Pireo): vedi Demostene, Contro Aristocrate, 77 sg.; Aristotele, Costituzione degli Ateniesi, 57, 3 sg.; Pausania, Descrizione della Grecia, I, 28, 11. Musti \& Beschi (1986, 311); MacDowell (1963, 82-84); Harris (2010, 127): «The aim behind these rules was obviously to prevent any contact between the killer and the land of Attica and thus avoid pollution».

45. Vedi Huxley $(1969,53)$. 


\section{RIASSUNTI}

Questo saggio prende in esame alcuni frammenti dell'Alcmeonide, poema epico di età tardoarcaica, con il fine di ricostruire alcuni momenti dell'azione che possono essere ricondotti alla figura di Alcmeone, il protagonista del poema. La vicenda dell'eroe presenta molti tratti in comune con quella di Oreste. Entrambi furono matricidi ed entrambi furono obbligati ad abbandonare il paese di origine e a vagare per lungo tempo in varie regioni della Grecia. La prima parte del saggio propone una ricostruzione degli eventi secondo la probabile versione seguita nell'Alcmeonide (momento del matricidio, esilio) e la pone a confronto con quella seguita nell'altro poema epico che includeva il medesimo tema: gli Epigoni. La seconda parte del saggio esamina l'arrivo di Alcmeone in Acarnania, alle foci dell'Acheloo. La narrazione di Tucidide (II, 102, 5), che probabilmente riprendeva la versione dell'Alcmeonide attraverso la mediazione di una tradizione locale, è di particolare interesse in quanto mostra i rischi della contaminazione e l'accortezza del personaggio che riesce a evitare i rischi del contagio.

Cet essai prend en examen quelques fragments de l'Alcméonide, poème épique de l'époque archaïque tardive, afin d'analyser des éléments qui peuvent être reconduits au personnage d'Alcméon, le protagoniste du poème. Ce récit présente beaucoup de traits communs avec celui d'Oreste. Tous les deux furent matricides et tous les deux furent obligés à quitter leurs pays d'origine et à errer longtemps dans plusieurs régions de la Grèce. La première partie de l'essai propose une reconstruction des épisodes ayant comme protagoniste Alcméon (matricide, exil) selon la version probable de l'Alcméonide et les compare avec l'autre poème épique qui racontait les mêmes faits: les Épigones. La deuxième partie analyse l'arrivée d'Alcméon en Acarnanie, à l'embouchure de l'Acheloos. Le récit de Thucydide (II, 102,5), probablement fondé sur une tradition locale s'inspirant de l'Alcméonide, est particulièrement intéressant car il montre la sagacité du personnage qui réussit à éviter le risque de la contamination.

This essay examines fragments of the Alcmeonis, an epic poem from the late archaic age, with the purpose of reconstructing some features of this work with particular reference to the main character Alcmeon. In many aspects, the story is similar to that of Orestes as reported by ancient authors. Both heroes were obliged to commit matricide, and both were obliged to leave their country and wander for a long time throughout Greece. The initial part of the study aims at reconstructing some moments from the story as it was probably narrated in the Alcmeonis, and proposes a comparison with the plot of the Epigoni. The latter part focuses on Alcmeon's arrival in Acarnania and his settlement at the mouth of the Acheloos. Thucydides (II, 102,5) relates a story of particular interest. The traditional topic of the quest for a new land is common to Orestes and Alcmeon, but Thucydides' version, which was probably derived from the Alcmeonis through the mediation of a local storytelling, presents features which emphasize the intelligence of the character, and his capability in facing the risk of pollution.

\section{INDICE}

Mots-clés : cycle épique, Alcméon, Alcméonide, Oreste, Thucydide, contamination

Parole chiave : ciclo epico, Alcmeone, Alcmeonide, Oreste, Tucidide, contaminazione

Keywords : epic cycle, Alcmaon, Alcmeonis, Orestes, Thucydides, pollution 
AUTORE

\section{CARLO BRILLANTE}

Università di Siena, Centro Antropologia e Mondo Antico

carolus.venetiae@gmail.com 